\title{
Normal Force and Sag Resistance of Pipe Conveyor
}

\author{
Shuaiping Guo ${ }^{1,2^{*}}$, Wei Huang ${ }^{1,2}$ and Xuejun $\mathrm{Li}^{1}$
}

\begin{abstract}
Pipe belt conveyor is a new type of environmentally friendly and efficient bulk conveying equipment. In the design of the roller, the belt and the driving motor of pipe belt conveyor, the sag resistance is a key parameter. Meanwhile, the normal force between the conveyor belt and the roller group is the other important factor need be considered and has a great influence on the sag resistance. This paper analyzes a pipe belt conveyor with a diameter of $150 \mathrm{~mm}$ to study the calculation method of normal force. And the relationship between the normal force and the sag resistance is explored. Firstly, the normal force is decomposed into three components related to the forming force of belt, material gravity and belt gravity. So it can be expressed as a linear combination of these three quantities, and the coefficients of each component are obtained based on the dynamic analysis of belt-roller. The results show that the coefficient is mainly affected by the material filling rate, and is almost not affected by the distance between the rollers and the density of the material. The calculation method of the normal force is eventually obtained. Secondly, the normal force in the case of different material filling rates is tested by experiments, and the calculation method of the normal force is verified. Thirdly, the variation law of the sag resistance in the case of different roller group spacing and material filling rate is studied by the dynamic model. It is found that the roller group spacing and material filling rate affects the sag resistance by changing the normal force. There is a power function relationship between the sag resistance and the normal force. In the case of different roller group spacing and material filling rate, the relationship among the sag resistance and the normal force remains unchanged. This study results are of great significance to the design of pipe belt conveyor.
\end{abstract}

Keywords: Pipe conveyor, FEM, Normal force, Sag resistance

\section{Introduction}

The pipe conveyor is a new type of environmentally friendly and efficient bulk material conveying equipment, which has the characteristics of large load, long distance, large capacity and continuous operation. It is increasingly popular in mining, metallurgy, chemical, electric power, port and other industries $[1,2]$. The running resistance is a key parameter in the design of the pipe conveyor. The excessive running resistance leads to the increase of the motor power and the power consumption. In severe

\footnotetext{
*Correspondence: guoshuaiping@163.com

1 Engineering Research Center of Advanced Mining Equipment Ministry of Education, Hunan University of Science and Technology, Xiangtan 411201, China

Full list of author information is available at the end of the article
}

cases, the pipe conveyor is overloaded and the service life is greatly reduced. Hager [3] analyzed components of running resistance for ordinary conveyor with a length of one kilometre, and the sag resistance accounted for $61 \%$ of the total running resistance. Because the belt of pipe conveyor is pipe-shaped, the contact force between the roller and the belt is greater than ordinary conveyor. The sag resistance of pipe conveyor accounts for a greater proportion of total resistance. Currently, in the design of the pipe conveyor, the sag resistance is calculated by product of normal force of belt-rollers and the sag resistance coefficient. The normal force is generated by the forming force of belt, belt gravity and material gravity on roller [4]. And the normal force of roller group often are calculated by taking the sum of material gravity, belt gravity and the forming force of belt between the two 
roller groups. The normal force obtained by this method will have a large error. Moreover, when calculating the sag resistance of pipe conveyors, the sag resistance coefficient used is considered to be equal to that for ordinary belts. This makes it impossible to calculate the sag resistance accurately. Considering the difference of working principle between the two conveyor types, a more reasonable calculation method of the normal force and sag resistance of pipe conveyor should be studied.

For the normal force of pipe conveyor, Molnár et al. [58] presented design and verification of regression models for prediction of pipe conveyor belt normal forces on rollers. Zamiralova et al. [9] presented an experimental study to investigate the influence of various factors on the normal force of the roller, such as pipe diameter, belt width, transverse bending stiffness, line mass and the position of the belt overlap. Zheng et al. [10] proposed FEM/DEM coupling model for simulating pipe conveyor systems to investigate the fundamental contact force acting between conveying belt and structural components. Bin et al. [11] established a rigid-flexible coupling model of belt and rollers for the pipe conveyor to solve the normal force in the case of the typical conditions of uniform speed and uniform acceleration. Michalik et al. [12] designed a computer integrated system for the evaluation of the normal force on rollers and the strains at different locations of a belt. Guo et al. [13, 14] studied the influence of diameter of pipe-shaped belt on normal force, and an optimized pipe diameter is obtained. Stehlikova et al. [15] analysed relationship among the tension forces, asymmetrical tensioning and filling rate in the crosssectional area of the piped belt. Lech Gładysiewicz et al. [16] measured loads on belt conveyor rollers in operation under actual conditions. Wang et al. $[17,18]$ studied the magnetic properties of the permanent magnet magnetic pipe conveyor belt system. An experimental device with adjustable diameter roller group was designed to test the dynamic pressure of the roller group. However, most of the studies above only analyzed the influence of different factors on static normal force. There were few studies on normal force based on the dynamic model and no trustworthy calculation method of the roller normal force. Hence, it is of great significance to research the influence of the material factor, the forming force of belt and the belt gravity on the normal force, and to obtain a trustworthy calculation method of the normal force.

For the sag resistance, Jonkers [19] derived the calculation formula of the sag resistance. Qin [20] provided a method to calculate the sag rolling resistance. O'Shea [21] analyzed different test methods of sag resistance. Rudolphi [22] used a one-dimensional Winkler foundation and a generalized viscoelastic Maxwell solid model of the belt backing material to determine the resistance of a conveyor belt over rollers. Reicks et al. [23] used two theoretical approaches to predict the loss for belt cover rubber. The results of these calculations are compared with the results of measurement, and important factors affected the accuracy of these prediction methods were offered. Leonardo and Santos [24] designed a low-cost measuring method for sag resistance of pipe conveyor. Yan [25] studied indentation rolling resistance of belt conveyor based on Hertz contact theory compared with one-dimensional Winkler foundation. Jayne [26] introduced a dielectric energy loss model which uses dielectric methods to measure the viscoelastic material properties of the bottom cover of conveyor belt. Robinson [27] established a spherical indentation into a generalized Maxwell backing. The indentation rolling resistance of spherically profiled idler rolls was studied. Dutta Sudipta [28] designed a table top experimental setup for electrical contact resistance measurement during indentation. Nicola Menga [29] studied the indentation rolling resistance in belt conveyors based on a model for the viscoelastic friction. Javier [30] presented the knife-edge-equivalent contact constraint method to analyses the two-point wheel-rail contact scenario. Zhang [31] tested the friction coefficient of belt conveyor under various working conditions.

However, most of the research on the sag resistance is about to ordinary belt conveyors, and these studies can't provide straight guidance for practical engineering design of the pipe conveyor. It is important to study the variation of the sag resistance in the case of different factors and research the relationship between the normal force and sag resistance, which can provide an important basis for the design of the sag resistance.

In this paper, a certain type of pipe conveyor is studied. Firstly, the normal force is decomposed into three components related to the forming force of belt, material gravity, and belt gravity. So the normal force can be expressed as a linear combination of these three quantities. The coefficients of three components will be obtained based on the dynamic analysis of belt-roller and function fitting. The calculation formula of the normal force of each roller is eventually obtained, which will be verified by the experiments in the case of different material filling rates. And then, the variation law of the sag resistance in the case of different roller group spacing and material filling rate is studied. The relationship between the sag resistance and the normal force is obtained based on above analysis.

The current paper is organized as follows. The calculation method of the normal force is presented and verified by experiments in Section 2. The influence of different factors on sag resistance is presented in Section 3. Finally, 
concluding remarks and directions for future research are discussed in Section 4.

Influence of different factors on sag resistance.

\section{Calculation Method of the Normal Force}

\subsection{Theoretical Analysis of the Normal Force}

The regular hexagon roller group is considered. The arrangement of rollers is shown in Figure 1.

There are six rollers in a roller group. The normal force of the $i$ th roller $F_{i}$ can be decomposed into three components $F_{C i}, F_{b i}$ and $F_{m i}$, which are related to the forming force of belt, material gravity, and belt gravity respectively.

$$
F_{i}=F_{C i}+F_{b i}+F_{m i} .
$$

If the pipe-shaped belt is a regular circle with no overlapping parts, then the forming force of the belt on these six rollers are equal. Defined $F_{C}$ as

$$
F_{C}=F_{C 1}=F_{C 2}=F_{C 3}=F_{C 4}=F_{C 5}=F_{C 6} .
$$

$F_{C}$ is related to the properties of the belt and the roller groups spacing. It can be obtained by experimental measurement. It also can be calculated based on elastic mechanics theory and properties of the belt. As the spacing between the roller group increases, $F_{C}$ will also increase. But the relationship between them is not linear.

However, the pipe-shaped belt is not a regular circle, $F_{C i}$ are not equal. So defined

$$
F_{C i}=\xi_{i} F_{C} .
$$

The coefficient $\xi_{i}$ is undetermined. Since roller 2 is located at overlap of the belt, $F_{C 2}$ is greater than $F_{C i}$ of other rollers. But $F_{C i}$ of other five rollers below are approximately equal, so $F_{\mathrm{C}}$ is redefined as

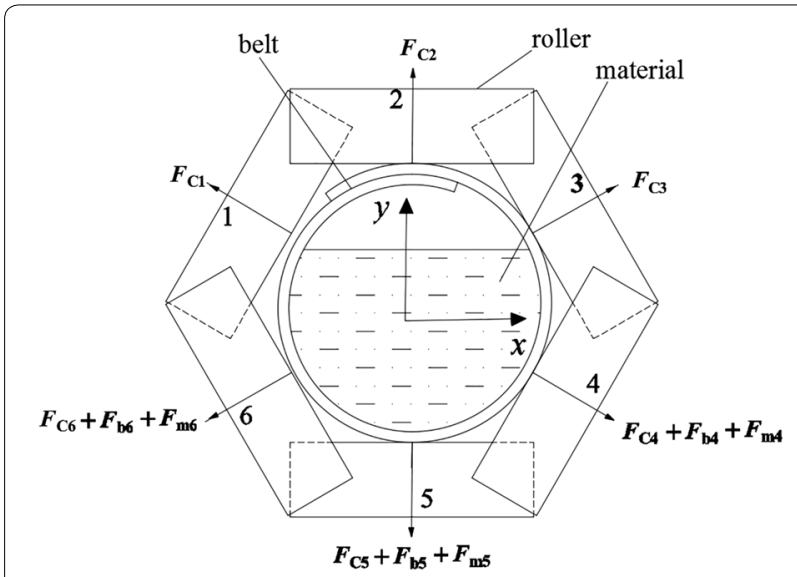

Figure 1 Force analysis of the roller

$$
F_{C}=\left(F_{C 1}+F_{C 3}+F_{C 4}+F_{C 5}+F_{C 6}\right) / 5 .
$$

And $F_{C i}$ of all rollers can still be represented by Eq. (3). All we have to do is determine the coefficients $\xi_{i}$.

In Eq. (1), $F_{b i}$ can be derived from the belt gravity $G_{b}$. Considering that the spatial position of each roller is different, $F_{b i}$ can be calculated by product of the belt gravity $G_{b}$ and the coefficient $\omega_{i}$ on each roller, which is

$$
F_{b i}=\omega_{i} G_{b} .
$$

The coefficient $\omega_{i}$ is undetermined, $G_{b}$ can be calculated according to the following formula

$$
G_{b}=B x t q g,
$$

where $B$ is the bandwidth, $x$ is the distance between each roller groups, $t$ is the thickness of belt, $g$ is the gravitational acceleration, $q$ is the density of the belt.

In Eq. (1), $F_{m i}$ can be derived from the material gravity $G_{m}$. It can be calculated as

$$
F_{m i}=\zeta_{i} G_{m},
$$

where $\zeta_{i}$ is the undetermined coefficient, and it will be affected by the material filling rate. The material gravity $G_{m}$ is

$$
G_{m}=\rho \pi\left(\frac{D}{2}\right)^{2} \psi x g
$$

where $\psi$ is the material filling rate. $\rho$ is the material density. $D$ is the diameter of pipe-shaped belt. Therefore, the normal force can be expressed as

$$
F_{i}=\xi_{i} F_{C}+\omega_{i} G_{b}+\zeta_{i} G_{m} .
$$

Based on the above analysis, the main research is to determine these three unknown coefficients.

Coefficients $\xi_{i}, \omega_{i}$ are affected by overlapping of the pipe-shaped belt. The overlapping is generally located at the top roller. The two coefficients can be determined when position and shape of overlapping are given. The coefficient $\zeta_{i}$ is mainly affected by $\psi$. This is because the pipe is round. As the material in the pipe increases, although $G_{m}$ increases linearly, the changing law of coefficient $\zeta_{i}$ is very complicated.

Hence, the dynamics model of belt-roller will be established, and the calculation formulas of the above three coefficients will be derived based on the dynamic simulation. And the material filling rate $\psi$ will be considered as an independent variable. 


\subsection{Influence of Material Filling Rate on Normal Force}

This part is based on finite element method, and the dynamic model of belt and rollers is established by ANSYS 17.0. The specific process is as follows.

Step 1: The geometric model of the pipe-shaped belt and rollers is established as shown in Figure 2. The element type of the belt and rollers is SOLID185.

Step 2: Established the contact pair of belt and rollers. Then, degrees of freedom UX and UY of belt's end face are constrained under the coordinate system as shown in Figure 1. The degrees of freedom of rollers are fixed. And then apply the acceleration due to the gravity. Finally, the model is solved to obtain the stable contact between the belt and rollers under the gravity.

Step 3: Loading torque on both sides of the belt to simulate the forming force of pipe-shaped belt, the gravity of material on the inner surface of belt is loaded according to Ref. [32]. After solving, the stable contact of belt and rollers in above conditions is obtained.

Step 4: Unfreeze the rotation degrees of freedom of the roller along the axis, and set a speed of $1 \mathrm{~m} / \mathrm{s}$ to the belt to make it move at a uniform speed. After solving, the dynamic contact force of the belt and rollers could be obtained.

The pipe-shaped belt will expand if the material filling rate $\psi>80 \%$, so $\psi \leq 80 \%$ generally in the engineering design. In order to study the normal force in the case of different filling rate, the dynamics model of beltrollers with the material filling rate $\psi$ of $0,10 \%, 20 \%, \ldots$, $80 \%$ is established. The material transported of the pipe conveyor is generally coal, ore, etc., and their density is between $1 \mathrm{t} / \mathrm{m}^{3}$ and $3 \mathrm{t} / \mathrm{m}^{3}$. The material density in experiment is $2.1 \mathrm{t} / \mathrm{m}^{3}$, so the density of dynamics model $\rho=2.1 \mathrm{t} / \mathrm{m}^{3}$ for easy comparison. The speed of the belt $v$ is $1 \mathrm{~m} / \mathrm{s}$. And the spacing between two adjacent roller groups $x$ is $1.2 \mathrm{~m}$. The dynamic model of belt-rollers is shown in Figure 2. In order to reduce the impact of the extension of the belt and material, the middle section in Figure 1 is taken to be researched. The arrangement order of the rollers is shown as Figure 1.

We will concern the average value of dynamic normal force because the engineering design mainly focuses on it. The average of dynamic normal force in $1 \mathrm{~s}$ is obtained by the dynamics model. $G_{m}$ in the case of different $\psi$ is calculated by Eq. (8). Table 1 lists the value of normal force and $G_{m}$. The variation curve of the normal force with filling rate is shown in Figure 3.

It should be noted that $G_{m}(\psi)$ in Table 1 represents the $G_{m}$ value at different $\psi$. And in the following, when $\psi$ appears in parentheses, it also represents the physical quantity is a function of $\psi$. It can be seen from Figure 3 that $F_{2}$ decreases slowly with the increase of $\psi . F_{1}$ and $F_{3}$ is hardly affected by $\psi . F_{4}, F_{5}$ and $F_{6}$ increases with the increase of $\psi$, and $F_{5}$ is the fastest one.

Subtracting $F_{i}(0)$ from $F_{i}(\psi), F_{m i}(\psi)$ can be obtained, which is one of the components of the normal force

$$
F_{m i}(\psi)=F_{i}(\psi)-F_{i}(0) .
$$

And the coefficients $\zeta_{i}$ in the case of different $\psi$ can be presented as

$$
\zeta_{i}(\psi)=F_{m i}(\psi) / G_{m}(\psi) .
$$

$\zeta_{i}(\psi)$ can be obtained according to Table 2 and Eqs. (10), (11). For roller 1 and roller 3 ,

$$
\left\{\begin{array}{l}
\zeta_{1}(\psi)=F_{m 1}(\psi) / G_{m}(\psi) \approx 0, \\
\zeta_{3}(\psi)=F_{m 3}(\psi) / G_{m}(\psi) \approx 0 .
\end{array}\right.
$$

For roller 2,

$$
\zeta_{2}(\psi)=F_{m 2}(\psi) / G_{m}(\psi)=-0.08 .
$$

$F_{m 2}$ decreases as increases of the material filling rate. For roller 4, roller 5 and roller $6, F_{m i}(\psi), G_{m}(\psi)$ and $\zeta_{i}(\psi)$ can be obtained according to Table 1 and Eqs. (10), (11). The above data are listed in Table 2.

It can be seen from Table 2 that the coefficients $\zeta_{i}(\psi)$ of three rollers below have a small fluctuation. Especially when $\psi$ is greater than $50 \%$, the range of $\zeta_{4}(\psi)$ is about $33.1 \%-34.6 \%$, the range of $\zeta_{5}(\psi)$ is about $58.7 \%-60.6 \%$, and the range of $\zeta_{6}(\psi)$ is about

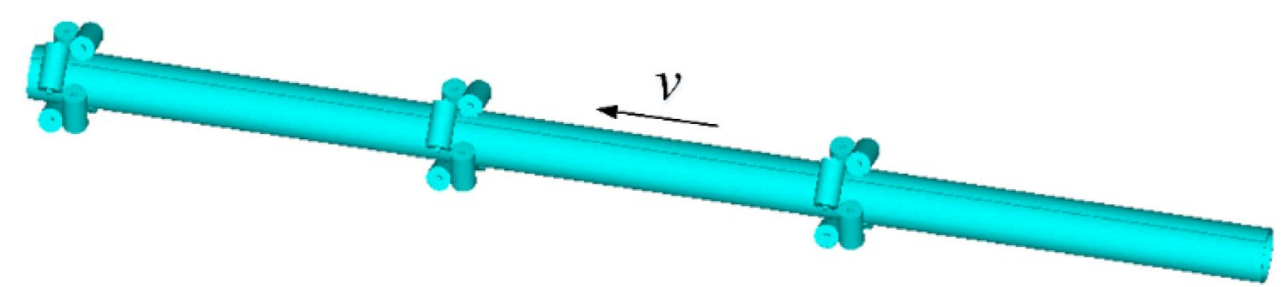

Figure 2 The dynamic model of belt-rollers 
Table 1 The normal force in the case of different material filling rates

\begin{tabular}{|c|c|c|c|c|c|c|c|}
\hline \multirow[t]{2}{*}{ Filling rate $\psi(\%)$} & \multicolumn{6}{|c|}{ The roller contact force $F_{i}(\mathrm{~N})$} & \multirow{2}{*}{$\begin{array}{l}G_{m}(\psi) \\
(\mathrm{N})\end{array}$} \\
\hline & $i=1$ & $i=2$ & $i=3$ & $i=4$ & $i=5$ & $i=6$ & \\
\hline 0 & 47.2 & 61.9 & 58.1 & 67 & 82.1 & 74.2 & 0 \\
\hline 10 & 45.5 & 58.3 & 56.8 & 79.7 & 108.5 & 86.1 & 44.5 \\
\hline 20 & 44.5 & 53.3 & 55.1 & 94.9 & 139 & 102.4 & 89.0 \\
\hline 30 & 44.9 & 49.1 & 54.2 & 111 & 169 & 122 & 133.5 \\
\hline 40 & 44.6 & 45.7 & 54.1 & 125.5 & 192.9 & 137 & 178.0 \\
\hline 50 & 46.3 & 42.4 & 53.8 & 140.6 & 217 & 150.8 & 222.5 \\
\hline 60 & 47.5 & 38.8 & 56.3 & 156 & 241 & 165.4 & 267.1 \\
\hline 70 & 48.1 & 35.9 & 58.3 & 174.8 & 266.3 & 179.7 & 311.0 \\
\hline 80 & 50.4 & 32.6 & 61.5 & 185.8 & 287.7 & 194.9 & 356.1 \\
\hline
\end{tabular}

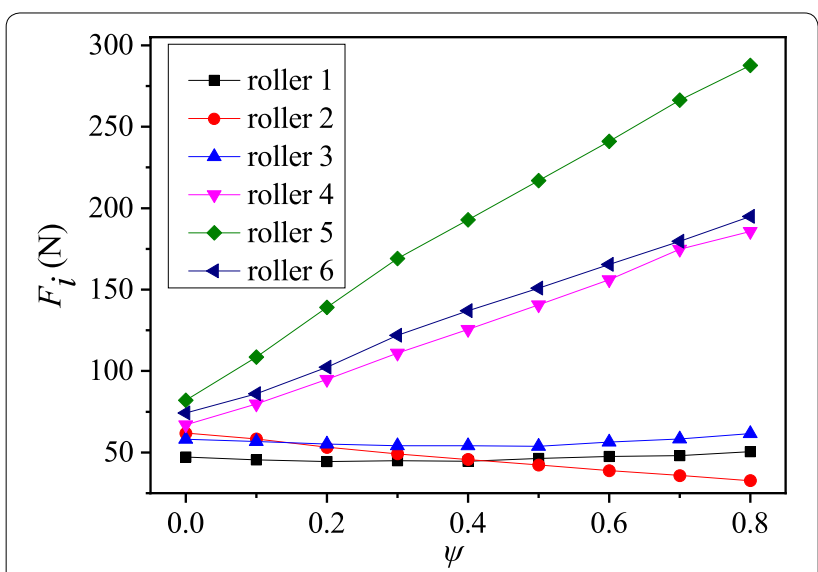

Figure 3 Variation curve of the normal force with filling rate

$33.8 \%-34.4 \%$. It can be considered that the coefficients are approximately constant at this time.

Therefore, when $\psi$ is less than $50 \%$, the relationship between the coefficients $\zeta_{i}(\psi)$ for $i=4,5,6$ and $\psi$ can be fitted by data of Table 2 , that is

$$
\left\{\begin{array}{l}
\zeta_{4}(\psi)=-0.50 \psi^{2}+0.41 \psi+0.25, \\
\zeta_{5}(\psi)=-1.18 \psi^{2}+0.71 \psi+0.54, \\
\zeta_{6}(\psi)=-1.17 \psi^{2}+0.89 \psi+0.19
\end{array}\right.
$$

When $\psi$ is greater than $50 \%$, the relationship between $\zeta_{i}(\psi)$ for $i=4,5,6$ and $\psi$ can be fitted by a constant value function, that is,

$$
\left\{\begin{array}{l}
\zeta_{4}(\psi)=0.33 \\
\zeta_{5}(\psi)=0.60 \\
\zeta_{6}(\psi)=0.34
\end{array}\right.
$$

Figure 4 shows the fitting function and its error of the coefficients $\zeta_{i}(\psi)$ of three rollers below. Among them, the points are the original data of $\zeta_{i}(\psi)$, the solid lines are the fitting functions of $\zeta_{i}(\psi)$, and the dotted lines are the fitting errors of $\zeta_{i}(\psi)$.

It can be seen from Figure 4 that the error of the fitting function $\zeta_{i}(\psi)$ is small, and the maximum error is no more than $2 \%$. Hence, the calculation formula of the material gravity component $F_{m i}$ can be obtained based on above analysis. $F_{m i}$ of the upper three rollers is

\begin{tabular}{|c|c|c|c|c|c|c|c|}
\hline \multirow[t]{2}{*}{$\psi(\%)$} & \multicolumn{3}{|c|}{$F_{m i}(\psi)(\mathrm{N})$} & \multicolumn{3}{|c|}{$\zeta_{i}(\psi)(\%)$} & \multirow{2}{*}{$\begin{array}{l}G_{m}(\psi) \\
(\mathrm{N})\end{array}$} \\
\hline & $i=4$ & $i=5$ & $i=6$ & $i=4$ & $i=5$ & $i=6$ & \\
\hline 10 & 12.7 & 26.4 & 11.9 & 28.5 & 59.3 & 26.7 & 44.5 \\
\hline 20 & 27.9 & 56.9 & 28.2 & 31.3 & 63.9 & 31.7 & 89.0 \\
\hline 30 & 44.0 & 86.9 & 47.8 & 33.0 & 65.1 & 35.8 & 133.5 \\
\hline 40 & 58.5 & 110.8 & 62.8 & 32.9 & 62.2 & 35.3 & 178.0 \\
\hline 50 & 73.6 & 134.9 & 76.6 & 33.1 & 60.6 & 34.4 & 222.5 \\
\hline 60 & 89.0 & 158.9 & 91.2 & 33.3 & 59.4 & 34.1 & 267.1 \\
\hline 70 & 107.8 & 185.2 & 105.5 & 34.6 & 59.5 & 33.9 & 311.0 \\
\hline 80 & 118.8 & 205.6 & 120.7 & 33.4 & 58.7 & 33.8 & 356.1 \\
\hline
\end{tabular}

Table 2 Value of $F_{m i}(\psi), G_{m}(\psi)$ and $\zeta_{i}(\psi)$ in the case of different material filling rates 


$$
\left\{\begin{array}{l}
F_{m 1}=F_{m 3}=0, \\
F_{m 2}=\zeta_{2} G_{m}=-0.08 G_{m} .
\end{array}\right.
$$

When $\psi<50 \%, F_{m i}$ of the below three rollers is

$$
\left\{\begin{array}{l}
F_{m 4}=\zeta_{4} G_{m}=\left(-0.50 \psi^{2}+0.41 \psi+0.25\right) G_{m}, \\
F_{m 5}=\zeta_{5} G_{m}=\left(-1.18 \psi^{2}+0.71 \psi+0.54\right) G_{m}, \\
F_{m 6}=\zeta_{6} G_{m}=\left(-1.17 \psi^{2}+0.89 \psi+0.19\right) G_{m} .
\end{array}\right.
$$

Meanwhile, when $\psi \geq 50 \%$,

$$
\left\{\begin{array}{l}
F_{m 4}=\zeta_{4} G_{m}=0.33 G_{m} \\
F_{m 5}=\zeta_{5} G_{m}=0.60 G_{m} \\
F_{m 6}=\zeta_{6} G_{m}=0.34 G_{m} .
\end{array}\right.
$$

Based on the above research, we can also find that when the filling rate $\psi$ is determined, the change in material density $\rho$ will also cause the change in normal force. This is because changes in $\rho$ will only cause changes in $G_{m}$ in the formula, and hardly affect the coefficients $\zeta_{i}$. Meanwhile, with the spacing $x$ between the roller groups changes, the essence of it is also to change the gravity of the materials by each roller groups. And it also hardly affects the coefficients $\zeta_{i}$. Therefore, $F_{m i}(\psi)$ at different $\psi, \rho$, and $x$ can be calculated using Eqs. (16)-(18).

\subsection{Influence of Belt Factors on the Normal Force}

The relationship between material gravity and the normal force is obtained, the influence of the forming force and the belt gravity on the normal force is still unclear. In order to determine the coefficients $\xi_{i}$, $\omega_{i}$, a dynamics model of belt-rollers is established which only considers the forming force of belt. $F_{C i}$, one of the components of $F_{i}$, can be obtained by the dynamics model above. We need to make some assumptions: 1 . the materials in the belt are evenly

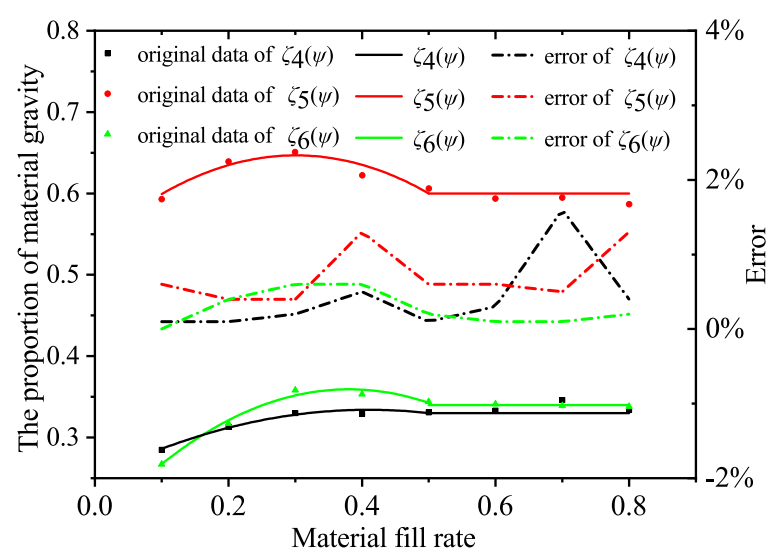

Figure 4 The fitting functions of $\zeta_{i}(\psi)$ and their errors distributed; 2. The tension force of the belt during the transportation process does not change much, and it has little effect on the normal force, and the tension force can be ignored. And $F_{b i}$ can be obtained as follows:

$$
F_{b i}=F_{i}(0)-F_{C i},
$$

where $F_{i}(0)$ is the normal force with no-load. The belt gravity $G_{b}=68.3 \mathrm{~N}$ is calculated by Eq. (6). The coefficient $\omega_{i}$ can be calculated by Eq. (5). Table 3 lists the value of $F_{i}(0), F_{C i}$. Then $F_{b i}$ and $\omega_{i}$ are calculated according to Eq. (19) and Eq. (5), respectively.

According to $F_{C i}$ in Table 3 and Eq. (4), we can obtain

$$
F_{C}=\left(F_{C 1}+F_{C 3}+F_{C 4}+F_{C 5}+F_{C 6}\right) / 5=53.2 \mathrm{~N},
$$

then,

$$
\left\{\begin{array}{l}
F_{C 1}=\xi_{1} F_{C}=0.95 F_{C}, \\
F_{C 2}=\xi_{2} F_{C}=1.49 F_{C}, \\
F_{C 3}=\xi_{3} F_{C}=1.15 F_{C}, \\
F_{C 4}=\xi_{4} F_{C}=0.93 F_{C}, \\
F_{C 5}=\xi_{5} F_{C}=0.91 F_{C}, \\
F_{C 6}=\xi_{6} F_{C}=1.06 F_{C} .
\end{array}\right.
$$

Concerning $F_{b i}$ in Table 3, it will be found that $F_{b i}$ are negative when $i=1,2$ and 3 . It means that, for the three rollers above, the belt gravity will balance a part of forming force. According to $F_{b i}$ in Table 3 and Eq. (5), we also can obtain

$$
\left\{\begin{array}{c}
F_{b 1}=\omega_{1} G_{b}=-0.05 G_{b}, \\
F_{b 2}=\omega_{2} G_{b}=-0.26 G_{b}, \\
F_{b 3}=\omega_{3} G_{b}=-0.05 G_{b}, \\
F_{b 4}=\omega_{4} G_{b}=0.25 G_{b}, \\
F_{b 5}=\omega_{5} G_{b}=0.49 G_{b}, \\
F_{b 6}=\omega_{6} G_{b}=0.26 G_{b} .
\end{array}\right.
$$

\subsection{Calculation Formula of the Normal Force}

According to $F_{C i}, F_{b i}$ and $F_{m i}$ obtained in Sections 2.2 and 2.3, Eqs. (16)-(18) and (21), (22), the calculation formula of normal force can be obtained eventually. For roller 1 , roller 2 and roller 3 , that is

$$
\left\{\begin{array}{l}
F_{1}=0.95 F_{C}-0.05 G_{b}, \\
F_{2}=1.49 F_{C}-0.26 G_{b}-0.08 G_{m}, \\
F_{3}=1.15 F_{C}-0.05 G_{b} .
\end{array}\right.
$$

For roller 4, roller 5 and roller 6 , the calculation formula of normal force could be presented by a piecewise function. When $\psi<50 \%$, the calculation formula is 
Table 3 Value of $F_{c i}$ and $F_{b i}$

\begin{tabular}{lllllll}
\hline $\boldsymbol{i}$ & $\mathbf{1}$ & $\mathbf{2}$ & $\mathbf{3}$ & $\mathbf{4}$ & $\mathbf{5}$ & $\mathbf{6}$ \\
\hline$F_{i}(0)(\mathrm{N})$ & 47.2 & 61.9 & 58.1 & 67 & 82.1 & 74.2 \\
$F_{C i}(\mathrm{~N})$ & 50.5 & 79.5 & 61.2 & 49.6 & 48.3 & 56.6 \\
$F_{b i}(\mathrm{~N})$ & -3.3 & -17.6 & -3.1 & 17.4 & 33.8 & 17.6 \\
$\omega_{i}$ & -0.05 & -0.26 & -0.05 & 0.25 & 0.49 & 0.26 \\
\hline
\end{tabular}

$$
\left\{\begin{array}{l}
F_{4}=0.93 F_{C}+0.25 G_{b}+\left(-0.50 \psi^{2}+0.41 \psi+0.25\right) G_{m}, \\
F_{5}=0.91 F_{C}+0.49 G_{b}+\left(-1.18 \psi^{2}+0.71 \psi+0.54\right) G_{m} \\
F_{6}=1.06 F_{C}+0.26 G_{b}+\left(-1.17 \psi^{2}+0.89 \psi+0.19\right) G_{m} .
\end{array}\right.
$$

When $\psi \geq 50 \%$, the normal force is

$$
\left\{\begin{array}{l}
F_{4}=0.93 F_{C}+0.25 G_{b}+0.33 G_{m}, \\
F_{5}=0.91 F_{C}+0.49 G_{b}+0.60 G_{m}, \\
F_{6}=1.06 F_{C}+0.26 G_{b}+0.34 G_{m} .
\end{array}\right.
$$

This calculation formula of normal force can calculate the normal force of roller with different $\psi, \rho$ and $x$. And only $\psi$ affects the coefficients $\zeta_{i}$ when $\psi<50 \%$. $\rho$ and $x$ will change $F_{C}, G_{b}$ and $G_{m}$, and they have little effect on coefficients in above formulas. The formulas can provide reference for the calculation of normal force under different working conditions.

\subsection{Test Verification of the Normal Force}

In order to verify the dynamics model and the calculation formula of normal force, the experimental device of the pipe conveyor is designed. The parameter of experimental device is similarity to the dynamics model, such as the thickness, the bandwidth and the rollers spacing. Figure 5 shows the experimental device of the pipe conveyor which is operated by a motor-driven.

The device of experimental data acquisition adopts 16-channel integrated acquisition and control module. It can realize synchronous acquisition of pressure signals, and the sampling frequency is set to $100 \mathrm{~Hz}$. The type of pressure sensor is JLBM-500. The upper and lower surfaces of the sensor are designed with studs and fixed by nuts. The sensitivity is $0.017(\mathrm{mV} / \mathrm{kg})$. Figure 6 shows the test schematic of the normal force. The pressure sensor is arranged between the roller brackets. Each roller has two pressure sensors. Transferring the signal collected by the sensor to the data acquisition card, then the signal is read by the test software of the master computer. Finally, the normal force can be obtained by sum of data of two pressure sensors.
Based on the experimental test, the normal force of the rollers with no-load and material filling rate of 30\%, 50\%, and $70 \%$ can be obtained. Table 4 lists the values of normal force from experiment.

The last three columns of Table 4 list the material gravity, the forming force of the belt, and the belt gravity. In order to verify the effectiveness of Eqs. (22)-(24), the calculation values are compared with the experimental value as shown in Figure 7 . The points are the experimental values of $F_{i}$, the solid lines represent the calculation formula of $F_{i}$.

It can be seen from Figure 7 that the accuracy of calculation formula is ideal. When $\psi=70 \%$, the relative error of roller 6 is the largest, which is $5.2 \%$. The errors of other rollers are all under $5 \%$. It is worth noting that there are measurement errors in the experimental measurement process, which also affects the comparison results. Hence, Eqs. (23)-(25) are effective to calculate normal force of each roller, which provides a theoretical basis for the roller structure design.

\section{Influence of Different Factors on Sag Resistance}

The sag resistance $P$ is the main source of running resistance, which is produced by the contact between the belt and the rollers. Based on experience in practical engineering applications, it can be found that the roller group spacing $x$ and material filling rate $\psi$ are the main factors to affect $P$. In this section, we will study the influence of $x$ and $\psi$ on $P$, and the relationship between $P$ and $F$. $F$ is the scalar sum of $F_{i}$ in a roller group.

\subsection{Influence of Roller Group Spacing on Sag Resistance} In order to research $P(x)$ in the case of different $x$, the dynamics model of belt-rollers with different roller group spacing is established. According to the requirements of drape of the belt, the roller group spacing $x$ should be between $0.8 \mathrm{~m}$ and $1.6 \mathrm{~m}$ for a pipe conveyor with a diameter of $150 \mathrm{~mm}$. Hence, in dynamic models, $x=0.8 \mathrm{~m}, 1.0 \mathrm{~m}, 1.2 \mathrm{~m}, 1.4 \mathrm{~m}$ and $1.6 \mathrm{~m}$, respectively. $\psi=70 \%$ and $\rho=2.1 \mathrm{t} / \mathrm{m}^{3}$ in the dynamic model. 


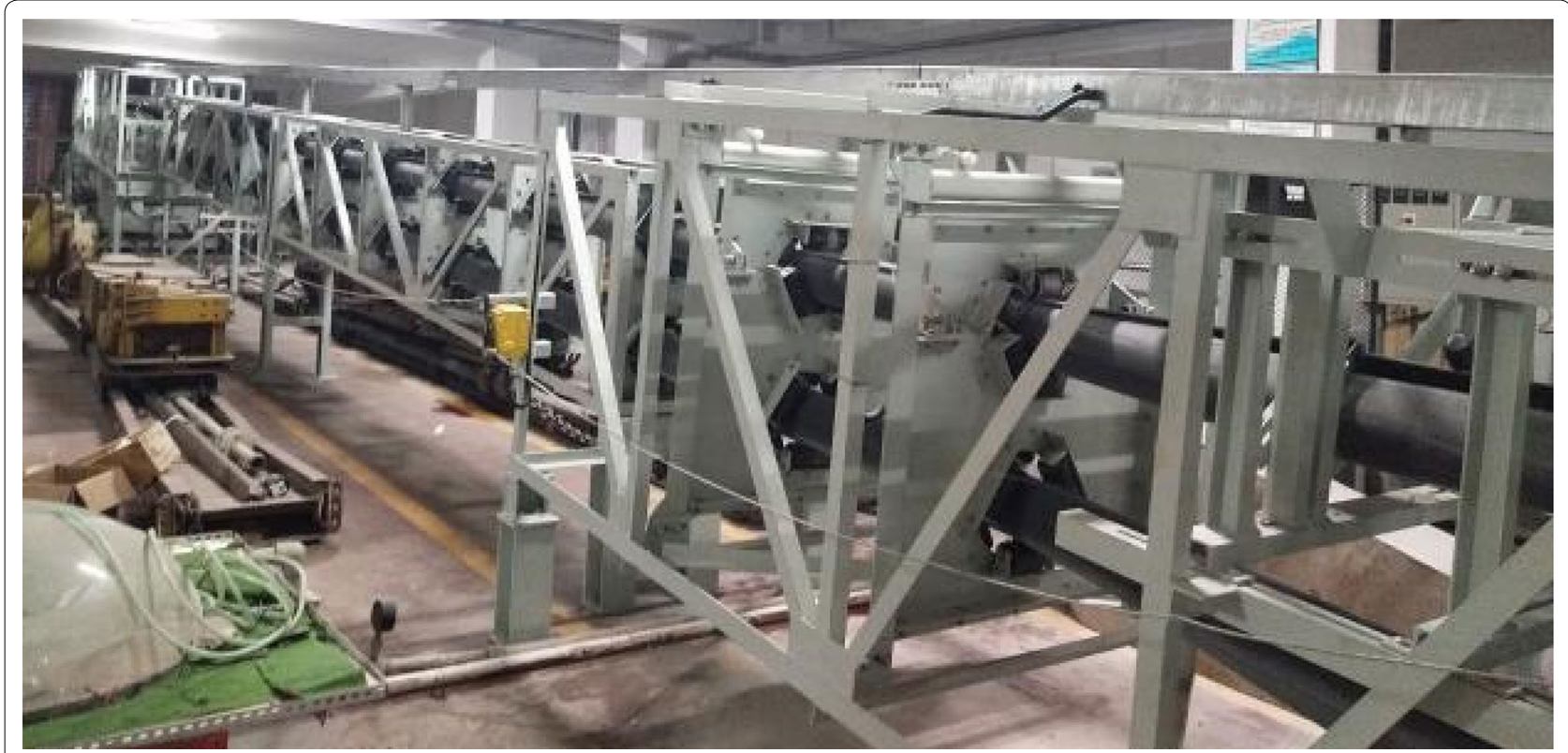

Figure 5 The experimental device of the pipe conveyor

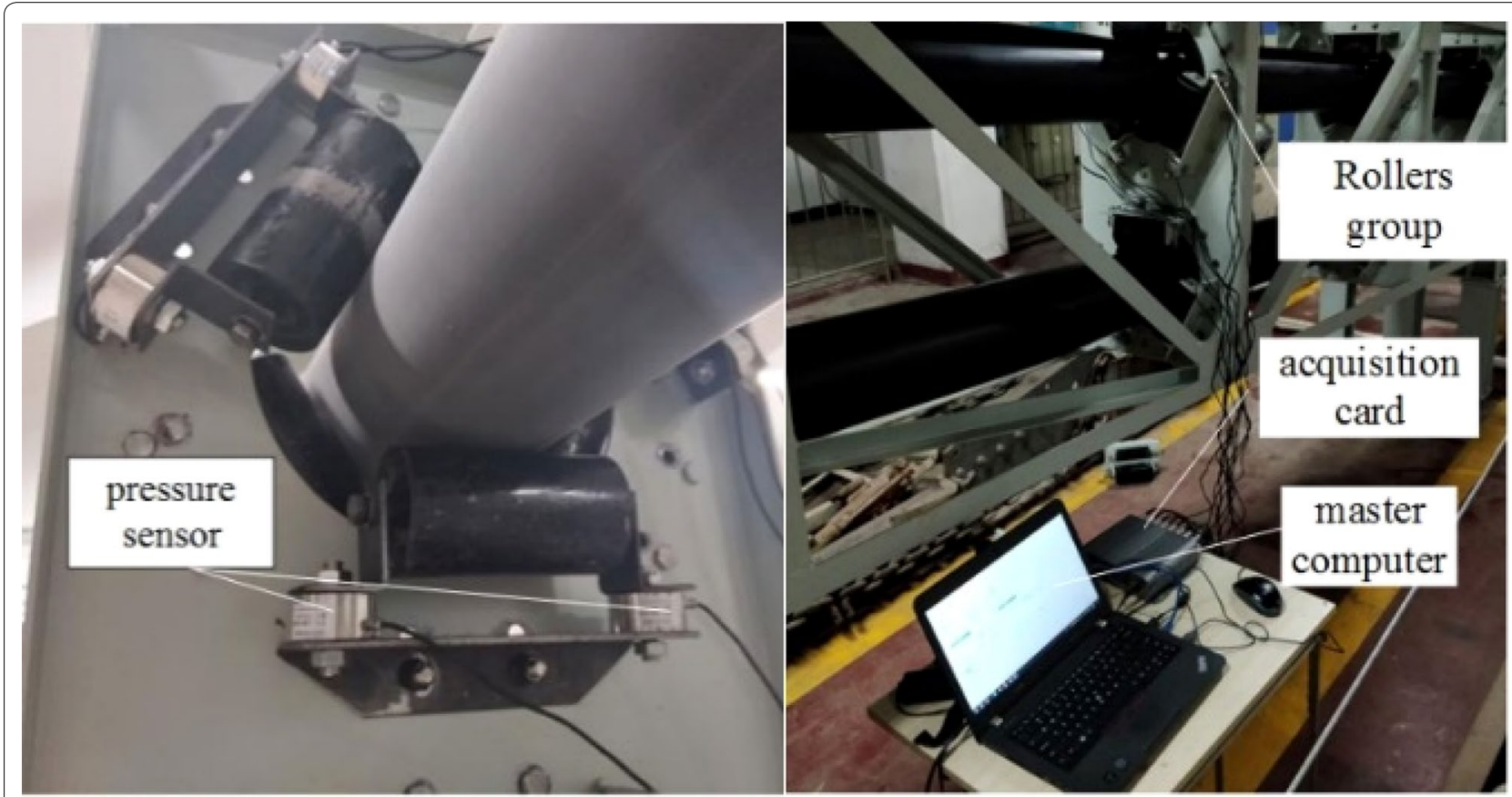

Figure 6 Test schematic of roller contact force

The value of $P(x)$ and $F(x)$ are obtained by the dynamics model. $F(x)$ is the sum of $F_{i}$ in the roller group and $P(x)$ is the sum of $P_{i} . P_{i}$ is the sag resistance at contact area between the belt and the $i$ th roller in the roller group. Table 5 lists the value of $P(x)$ and $F(x)$ from simulation. According to Table 5, a fitting function between $P(x)$ and $x$ can be obtained when $x=[0.8,1.6]$, that is 
Table 4 Roller normal force in the case of different $\psi$

\begin{tabular}{|c|c|c|c|c|c|c|c|c|c|}
\hline \multirow[t]{2}{*}{$\psi(\%)$} & \multicolumn{6}{|c|}{ The normal force $F_{i}(\mathrm{~N})$} & \multirow[t]{2}{*}{$F_{C}(\mathrm{~N})$} & \multirow[t]{2}{*}{$G_{b}(\mathrm{~N})$} & \multirow[t]{2}{*}{$G_{m}(\mathrm{~N})$} \\
\hline & $i=1$ & $i=2$ & $i=3$ & $i=4$ & $i=5$ & $i=6$ & & & \\
\hline 0 & 49.2 & 64.9 & 57.8 & 66.7 & 84.1 & 72.2 & 47.0 & 68.3 & 0.0 \\
\hline 30 & 46.7 & 51.6 & 50.5 & 108.1 & 172.5 & 112.8 & & & 133.5 \\
\hline 50 & 48.5 & 44.2 & 57.9 & 138.7 & 222.9 & 148.6 & & & 222.5 \\
\hline 70 & 55.2 & 33.3 & 57.1 & 159.3 & 270.4 & 167.9 & & & 311.0 \\
\hline
\end{tabular}

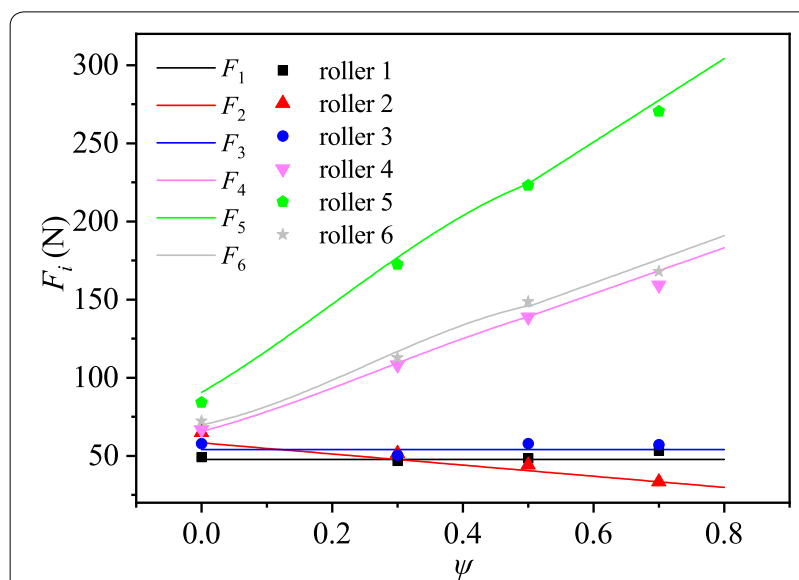

Figure 7 Comparison of the normal force between experiment and the calculation formula

$$
P(x)=27.2 x^{3.38}
$$

Figure 8 shows the value of sag resistance and its fitting function. It can be seen that the fitting effect is ideal and the fitting error is not more than $1 \%$. There is a power function relationship between $P(x)$ and $x$. When $x$ increases from $0.8 \mathrm{~m}$ to $1.6 \mathrm{~m}$, the value of $P(x)$ in a single roller group increases by 12.6 times. It indicates that the growth of sag resistance is extremely fast with roller group spacing increasing.

At this time, the relationship between the normal force $F(x)$ and sag resistance $P(x)$ is also very attractive. Hence, it can be obtained according to the data in Table 5 .

$$
P(x)=6 \times 10^{-7} F(x)^{2.76} .
$$

The comparison of the fitting function and original data is shown in Figure 9. It can be seen that the fitting error is not more than $1 \%$. And there is a power function relationship between $P(x)$ and $F(x)$. It is worth noting that the belt forming force $F_{c}$ and $x$ are non-linear by simultaneous Eqs. (26), (27), although $x$ has little effect on the coefficient $\xi_{i}$ in Eq. (5).
Table 5 The value of $P(x)$ and $F(x)$ in the case of different $x$

\begin{tabular}{lrrrrr}
\hline $\boldsymbol{x}(\mathbf{m})$ & \multicolumn{1}{c}{$\mathbf{0 . 8}$} & \multicolumn{1}{c}{$\mathbf{1 . 0}$} & $\mathbf{1 . 2}$ & \multicolumn{1}{c}{$\mathbf{1 . 4}$} & \multicolumn{1}{c}{$\mathbf{1 . 6}$} \\
\hline$F(x)(N)$ & 433.3 & 599.2 & 767.1 & 901.9 & 1027.6 \\
$P(x)(N)$ & 12.6 & 25.7 & 58.3 & 82.0 & 127.2 \\
\hline
\end{tabular}

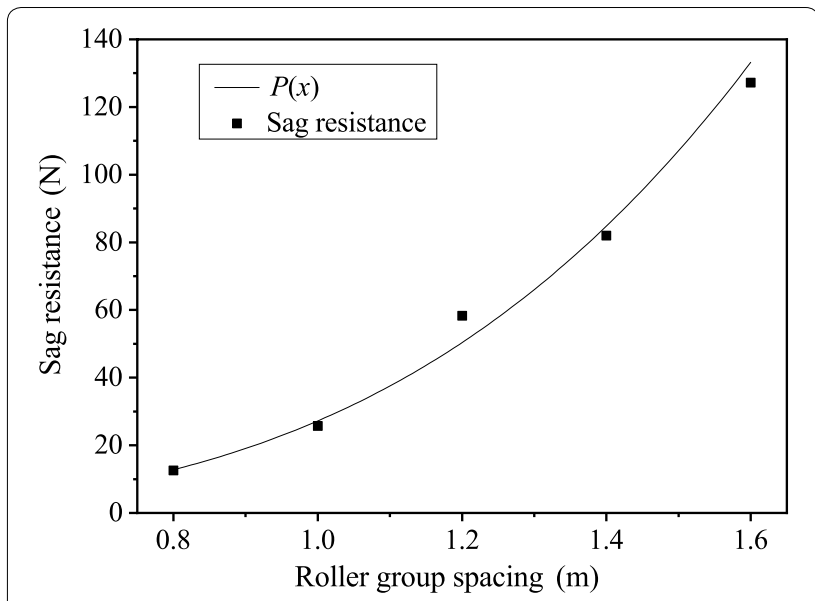

Figure 8 The sag resistance in the case of different rollers spacing and its fitting function

\subsection{Influence of Material Filling Rate on Sag Resistance}

In order to study the sag resistance in the case of different $\psi$, the value of $P(\psi)$ and $F(\psi)$ are obtained by the dynamics model with $\psi=0,10 \%, 20 \%, \ldots, 80 \%$. In simulation, only $\psi$ is the factor of change and $x$ is a constant. Table 6 lists the value of $P(\psi)$ and $F(\psi)$. Figure 10 shows the variation of $P(\psi)$.

It can be seen from Figure 10 that $P(\psi)$ increases as $\psi$ increases, and there is a power function relationship between $P(\psi)$ and $\psi$.

In order to further study the relationship between $P(\psi)$ and $F(\psi)$ in the case of the different material filling rate $\psi$, the function of $P(\psi)$ with respect to $F(\psi)$ is fitted according to the data in Table 6, which is

$$
P(\psi)=6 \times 10^{-7} F(\psi)^{2.76} .
$$


The maximum error of the fitting function is $1 \%$. And it can be known from Eq. (28) that there is a power function relationship between $P(\psi)$ and $F(\psi)$ in the case of different material filling rates $\psi$.

\subsection{Relationship between the Sag Resistance and the Normal Force}

According to above research in Sections 2.2, 2.3 and Eqs. (27), (28), $x$ and $\psi$ would affect the normal force $F$, then would further influence sag resistance $P$. And the relationship between $P$ and $F$ is the same in the case of different $x$ and $\psi$. There is a power function relationship between $P$ and $F$, that is

$$
P=6 \times 10^{-7} F^{2.76} \text {. }
$$

Based on the dynamics model, several sets of $P$ and $F$ values under different conditions were obtained and compared with the fitting function in Figure 11. The comprehensive error between the value of $P$ and fitting function error is about $2 \%$.

In Eq. (29), when $F$ increases from $400 \mathrm{~N}$ to $800 \mathrm{~N}, F$ increases by 2 times and $P$ increases by 7.11 times. It indicated that the sag resistance increases sharply with the increase of the normal force. In the design of the pipe conveyor, $F$ should be minimized, such as reducing $x$ and $\psi$, to reduce $P$.

According to the above analysis, the results show that changes in $x$ and $\psi$ essentially affect the normal force $F$, which further changes the sag resistance $P$. No matter how $x$ and $\psi$ changes, the relationship between $F$ and $P$ is consistent.

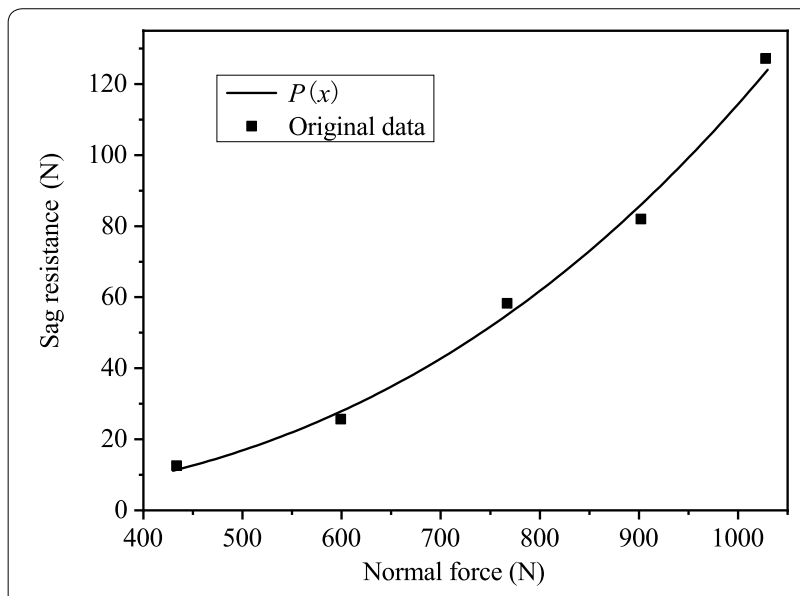

Figure 9 Relationship between sag resistance and normal force with different rollers spacing
Table 6 Value of $P(\psi)$ and $F(\psi)$ in the case of different material filling rate

\begin{tabular}{lll}
\hline $\boldsymbol{\psi ( \% )}$ & $\boldsymbol{F}(\boldsymbol{\psi})(\mathrm{N})$ & $P(\boldsymbol{\psi})(\mathrm{N})$ \\
\hline 0 & 390.5 & 9 \\
10 & 434.9 & 11.9 \\
20 & 489.2 & 15.1 \\
30 & 550.2 & 22.2 \\
40 & 599.8 & 30.2 \\
50 & 650.9 & 38.7 \\
60 & 705 & 48.2 \\
70 & 763.1 & 58.3 \\
80 & 812.9 & 68.2 \\
\hline
\end{tabular}

\section{Conclusions}

This paper proposes a calculation method of the normal force, analyzes variation of the sag resistance in case of different roller group spacing and material filling rate, and reveals the relationship between the sag resistance and the normal force. The following conclusions can be obtained from this paper.

(1) The normal force is decomposed into three components related to the forming force of belt, material gravity and belt gravity. The coefficients of three components are studied by the dynamics model of the belt and rollers. Then a calculation method of the normal force is obtained, in which the coefficient of material gravity is only related to the material filling rate.

(2) Based on experimental verification, the calculation method of the normal force can calculate the nor-

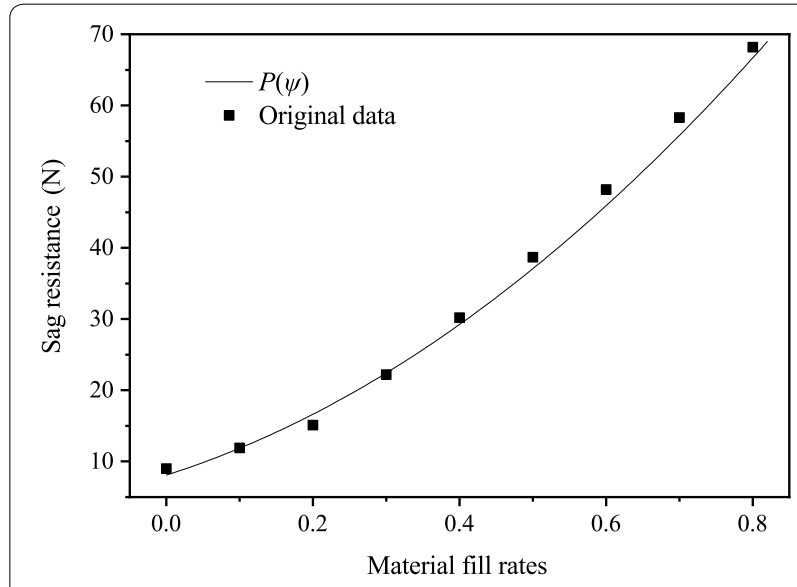

Figure $\mathbf{1 0}$ Variation of sag resistance in the case of different material filling rates 


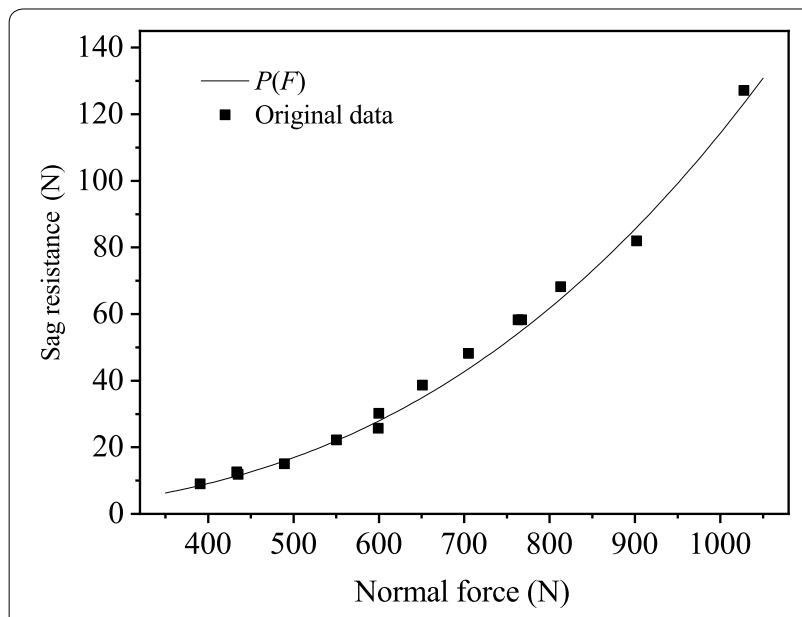

Figure 11 Relationship between the normal force and the sag resistance

mal force of each roller effectively, which provides a theoretical basis for the roller structure design.

(3) The roller group spacing and material filling rate would affect the normal force, then would further affect the sag resistance. There is a power function relationship between the sag resistance and the normal force of the roller group. The sag resistance can be calculated approximately according to this relationship and the normal force.

(4) The paper only research straight section of the pipe conveyor. However, in actual operation, a conveyor will turn and climb. It is significance to research the relationship between the normal force and sag resistance in case of different turning radius. This is what we need to study in the future.

\section{Acknowledgements}

Not applicable.

\section{Authors' Contributions}

SG and XL was in charge of the whole trial; SG and WH wrote the manuscript; WH completed the rectification experiment data collection; SG and WH undertook simulation analysis and data processing; SG has completed the revision of the manuscript rework and expert comments reply. All authors read and approved the final manuscript.

\section{Authors' Information}

Shuaiping Guo, born in 1987, is currently a lecturer at Hunan University of Science and Technology, Engineering Research Center of Advanced Mining Equipment Ministry of Education, China and Jiangsu Key Laboratory of Mine Mechanical and Electrical Equipment, China University of Mining and Technology. He received his doctor's degree on Hunan University, China, in 2015. His research interests include the dynamics, fault diagnosis and prognostic and engineering transportation and design. Tel: +86-13755004648.

Wei Huang, born in 1993, is currently a research assistant at Engineering Research Center of Advanced Mining Equipment Ministry of Education, Hunan University of Science and Technology, China. He received his master's degree from Hunan University of Science and Technology, China, in 2019. His research interests include the dynamics and Engineering transportation and design.

Xuejun Li, born in 1967, is currently a professor at Hunan University of Science and Technology, Engineering Research Center of Advanced Mining Equipment Ministry of Education, China. He received his doctor's degree on Central South University, China, in 2003. His research interests include the dynamics, fault diagnosis and prognostic.

\section{Funding}

Supported by National Natural Science Foundation of China (Grant No. 51705144), Hunan Provincial Science and Technology Major Project of China (Grant No. 2015GK1003), and Jiangsu Provincial Mine Electromechanical Equipment Key Laboratory Development Fund of China (Grant No. JSKL-MMEE-2018-2).

\section{Competing Interests}

The authors declare no competing financial interests.

\section{Author Details}

${ }^{1}$ Engineering Research Center of Advanced Mining Equipment Ministry of Education, Hunan University of Science and Technology, Xiangtan 411201, China. ${ }^{2}$ Jiangsu Key Laboratory of Mine Mechanical and Electrical Equipment, China University of Mining and Technology, Xuzhou 221116, China.

Received: 27 August 2019 Revised: 13 May 2020 Accepted: 28 May 2020 Published online: 12 June 2020

\section{References}

[1] P Staples. The history of pipe conveyors. Bulk Solids Handling, 2002, 22(3): 210-213.

[2] Y Wang, Q G Du, G Han. Environment -friendly continuous conveying equipment -The pipe conveyor. Journal of Mechanical Engineering, 2003, 39(1): 149-158. (in Chinese)

[3] M Hager, A Hintz. The energy saving-design of belts for long conveyor systems. Bulk Solids Handling, 1993, 13(4): 749-758.

[4] W G Song, H B Ji. Research progress and design calculation method of pipe conveyor. Chinese Journal of Engineering Design, 2018, 25(01): 1-11. (in Chinese)

[5] V Molnár, M Sabovčík. Static testing evaluation of pipe conveyor belt for different tensioning forces. Open Engineering, 2019, 9(1): 580-585.

[6] V Molnar, G Fedorko, B Stehlikova, et al. Influence of tension and release in piped conveyor belt on change of normal contact forces in hexagonal roller housing for pipe conveyor loaded with material. Measurement, 2016, 84: 21-31.

[7] V Molnár, G Fedorko, Lubor. Utilisation of measurements to predict the relationship between contact forces on the pipe conveyor idler rollers and the tension force of the conveyor belt. Measurement, 2019: 735-744.

[8] V Molnar, G Fedorko, S Honus, et al. Prediction of contact forces on idler rolls of a pipe conveyor idler housing for the needs of its online monitoring. Measurement, 2019: 177-184.

[9] M Zamiralova, G Lodewijks. Measurement of a pipe belt conveyor contact forces and cross section deformation by means of the six point pipe belt stiffness testing device. Measurement, 2015, 70: 232-246.

[10] Q J Zheng, M H Xu, KW Chu, et al. A coupled FEM/DEM model for pipe conveyor systems: Analysis of the contact forces on belt. Powder Technology, 2017: 480-489.

[11] G F Bin, W Q Zhang, X J Li, et al. Dynamic contact force analysis considering pipe conveyor belt elasticity. Journal of China Coal Society, 2017, 42(9): 2483-2490. (in Chinese)

[12] P Michalik, J Zajac. Using of computer integrated system for static tests of pipe conveyer belts. Proceedings of the 13th International Carpathian Control Conference (ICCC), High Tatras, Slovakia May 28-31, 2012: 480-485.

[13] Y Guo, S Wang, K Hu, et al. Optimizing the pipe diameter of the pipe belt conveyor based on discrete element method. 3D Research, 2016, 7(1).

[14] Guo Y, S Wang, K Hu, et al. Optimization and experimental study of transport section lateral pressure of pipe belt conveyor. Advanced Powder Technology, 2016, 27(4): 1318-1324. 
[15] B Stehlikova,V Molnar,G Fedorko, et al. Research about influence of the tension forces, asymmetrical tensioning and filling rate of pipe conveyor belt filled with the material on the contact forces of idler rolls in hexagonal idler housing. Measurement, 2020, 156: 107598.

[16] L Gładysiewicz, R Król, W Kisielewski. Measurements of loads on belt conveyor idlers operated in real conditions. Measurement, 2019, 134: 336-344.

[17] S Wang, D Li, Y Guo, et al. Research on magnetic model of low resistance permanent magnet pipe belt conveyor. 3D Research, 2016, 7(3): 23.

[18] SWang, D Li, K Hu, et al. Analysis and experimental study on pressure characteristics of supporting roller group of pipe belt conveyor. Shock and Vibration, 2019.

[19] C O Jonkers. The indentation rolling resistance of belt conveyors: A theoretical approach. In: Fordern und Heben, A Theoretical Approach, 1980, 30(4): 384-391.

[20] F Qin, Y Yu, T Rudolphi. Dynamic modeling of viscoelastic stress analysis in the case of moving loads. Proceedings of World Academy of Science Engineering \& Technology, 2011, 1(74): 226-233.

[21] J O'Shea, CWheeler, P Munzenberger, et al. The influence of viscoelastic property measurements on the predicted rolling resistance of belt conveyors. Journal of Applied Polymer Science, 2014, 131(18): 9170-9178.

[22] T J Rudolphi, A V Reicks. Viscoelastic indentation and resistance to motion of conveyor belts using a generalized maxwell model of the backing material. Rubber Chemistry and Technology, 2006, 79(2): 307-319.

[23] A Reicks, T Rudolphi, CWheeler. A comparison of calculated and measured indentation losses in rubber belt covers. Bulk Solids Handling, 2012, 32(3): 52-57.

[24] L e Santos, P Ribeiro, E Macêdo. Development of idler for measuring indentation rolling resistance in pipe conveyor belts. Measurement, 2020, 153: 107413.
[25] L Yan. Investigation on indentation rolling resistance of belt conveyor based on Hertz contact theory compared with one-dimensional Winkler foundation. Advances in Mechanical Engineering, 2018, 10(7): 1687814018783938.

[26] J O'Shea, C Wheeler. Dielectric relaxation studies of conveyor belt compounds to determine indentation rolling resistance. International Journal of Mechanics and Materials in Design, 2017, 13(4): 553-567.

[27] P Robinson, CWheeler. The indentation rolling resistance of spherically profiled idler rolls. International Journal of Mechanical Sciences, 2016, 106: 363-371.

[28] S Dutta, G Vikram, M Bobji, et al. Table top experimental setup for electrical contact resistance measurement during indentation. Measurement, 2020, 152: 107286.

[29] N Menga, F Bottiglione, G Carbone. The indentation rolling resistance in belt conveyors: A model for the viscoelastic friction. Lubricants, 2019, 7(7): 58.

[30] J Aceituno, P Urda, E Briales, et al. Analysis of the two-point wheel-rail contact scenario using the knife-edge-equivalent contact constraint method. Mechanism and Machine Theory, 2020, 148: 103803.

[31] D S Zhang, Z F Zhang, X H Wang. Study on friction coefficient test and operation energy consumption of belt conveyor. Chinese Mechanical Design and Research, 2019, 35 (01): 65-69. (in Chinese)

[32] Y Xu, G F Bin, G L Ye, et al. Research on limit conveying inclination in circular climbing section of pipe belt conveyor based on relative slip theory. Journal of China Coal Society, 2018, 43(11): 294-300. (in Chinese)

\section{Submit your manuscript to a SpringerOpen ${ }^{\circ}$ journal and benefit from:}

- Convenient online submission

- Rigorous peer review

- Open access: articles freely available online

- High visibility within the field

- Retaining the copyright to your article

Submit your next manuscript at $\boldsymbol{\Delta}$ springeropen.com 